\title{
Research on the Setting Method of Maintenance Equipment Supply Standard Based on Life Distribution
}

\author{
Chen Yu-kun ${ }^{1,}$, Gao $\mathrm{Qi}^{1,}$ a, Su Xiao-bo ${ }^{2, a}$ and Ning Hao-nan ${ }^{1, \text { a }}$ \\ ${ }^{1}$ Ordnance Engineering College, Shijiazhuang 050003, China \\ ${ }^{2}$ Mechanized Infantry Academy, Shijiazhuang 050003, China \\ aheianzhihou123@163.com
}

Keywords: maintenance equipment; life distribution; supply standard.

\begin{abstract}
Under the condition that maintenance equipment consumption data is insufficient in the initial support period, this article established development method of maintenance equipment supply standard of under exponential distribution, normal distribution and Waybill distribution respectively. And through the calculation of examples, the scientific nature of the method is verified. The conclusion can provide the theoretical basis for the development of the initial maintenance equipment supply standard.
\end{abstract}

\section{Introduction}

Initial maintenance equipment is used for repairing equipment in the initial support time before forming combat force. Because the equipment has just been in the stage of the column in this time, the information of consumption and fault of unit can hardly be collected. And the supply standards are difficult to develop. At present, the methods of forecasting the demand for maintenance equipment in the initial support time are mainly similarity analysis method, engineering analysis method, fuzzy comprehensive evaluation method, expert decision method, fund analogy method, case analysis method and so on. But these methods lack sufficient using data and scientific evidence, and also are different from the actual demand greatly. Besides, the above setting method of supply standard is based on the calculation of single unit, which lack overall coordination and optimization. So it is very difficult to guarantee the forecasting quantity demand for maintenance equipment reasonable. Therefore, this paper puts forward a method to establish the model for setting supply standard of maintenance equipment based on life distribution.

\section{The model of developing maintenance equipment supply standards}

\subsection{Basic model}

The model of developing maintenance equipment supply standards is to calculate the minimum number of maintenance equipment based on the working time of unit, the life distribution of the unit and the support probability. The problem can be described as follows

$$
S_{\text {min }}=\min \left\{S \mid \sum_{i=0}^{S} P(i) \geq P_{g}\right\}=\min \{S \mid P(i \leq S) \geq P\}
$$

Where,

$P_{g}$ — the support probability according to the provisions.

The steps of calculating the demand for maintenance equipment are as follows:

The first step: order $S=0$;

The second step: through the support probability of maintenance equipment, calculate $P(k \leq S)$, and calculate $P$;

The third step: if $P \geq P_{g}$, the algorithm is over, if $P<P_{g}$, the provision is to go to the fourth step;

The fourth step: order $S=S+1$, go to the second step. 
Therefore, the core of the problem is to solve the support probability of the maintenance equipment under the given conditions. And then, the forecasting model of demand for maintenance equipment under conditions of single unit and multiple unit are established respectively.

\subsection{Assumptions}

(1)the performance of the equipment does not change significantly with the time during the initial maintenance equipment support period, that is, it is not because of the failure of the equipment performance that to cause unit fault.

(2) Maintenance equipment is not invalid during storage.

(3) Due to the short installation time, the number of grade maintenance is zero.

(4) the way of repairing equipment is replacement, so do not consider whether the unit can be repaired;

(5) The time that replacing fault unit is negligible.

\subsection{The forecasting model of demand under conditions of single unit}

For a single unit, to calculate the support probability of the maintenance equipment, we can define an update process. Order the life of the $i$ th unit in system is $T_{i}(i-1,2, \cdots, n)$. Then the continuous working time of the system is a sequence as follows when the number of maintenance equipment in need is $n$.

$$
X_{n}=T_{1}+T_{2}+\cdots+T_{n-1}+T_{n}=X_{n-1}+T_{n}
$$

So the failure times of unit in $T_{X}$ is $k(k=0,1, \cdots, n)$. According to the theory of update process, the probability that the number of maintenance equipment in need is $k$ can be calculate.

$$
P(k)=F^{(k)}\left(T_{X}\right)-F^{(k+1)}\left(T_{X}\right)
$$

Where,

$P(k)$ - probability that the number of maintenance equipment required is $k$;

$T_{X}$ — - supply time of maintenance equipment;

$F^{(k)}\left(T_{X}\right)-k$ fold convolution function of $F(t)$.

The formula for calculating the support probability of the maintenance equipment is

$$
P(k \leq N)=\sum_{k=0}^{N} P(k)=\sum_{k=0}^{N}\left[F^{(k)}\left(T_{X}\right)-F^{(k+1)}\left(T_{X}\right)\right]
$$

Where,

$N$ - The Number of required maintenance equipment;

$P(k \leq N)$ — - the support probability of the maintenance equipment.

(1) The life of unit obeys the exponential distribution

When the unit is subject to an exponential distribution with a failure rate of $\lambda$, the fault density function $f(t)$ and the cumulative failure distribution function $F(t)$ of the unit can be calculated as follows.

$$
\begin{array}{r}
f(t)=\lambda \exp (-\lambda t) \\
F(t)=1-\exp (-\lambda t)
\end{array}
$$

Then the formula of $k$ fold convolution function of $F(t)$ in $T_{X}$ is

$$
\begin{gathered}
F^{(k)}\left(T_{X}\right)=1-\sum_{i=0}^{k-1} \frac{\left(\lambda T_{X}\right)^{i}}{i !} \exp \left(-\lambda T_{X}\right) \\
F^{(k+1)}\left(T_{X}\right)=1-\sum_{i=0}^{k} \frac{\left(\lambda T_{X}\right)^{i}}{i !} \exp \left(-\lambda T_{X}\right)
\end{gathered}
$$

By formula (3) , $P(k)$ can be calculated as follows. 


$$
P(k)=\frac{\left(\lambda T_{X}\right)^{k}}{k !} \exp \left(-\lambda T_{X}\right)
$$

By formula (4), $P(k \leq N)$ can be calculated as follows.

$$
P(k \leq N)=\sum_{k=0}^{N} \frac{\left(\lambda T_{X}\right)^{k}}{k !} \exp \left(-\lambda T_{X}\right)
$$

(2) The life of unit is in normal distribution

When the life of unit is in normal distribution, the fault density function and the cumulative failure distribution function of the unit can be calculated as follows.

$$
\begin{array}{r}
f(t)=\frac{1}{\sigma \sqrt{2 \pi}} \exp \left[-\frac{(t-\mu)^{2}}{2 \sigma^{2}}\right] \\
F(t)=\int_{0}^{t} \frac{1}{\sigma \sqrt{2 \pi}} \exp \left[-\frac{(x-\mu)^{2}}{2 \sigma^{2}}\right] \mathrm{d} x
\end{array}
$$

Where,

$\mu-$ Mean value of life of unit;

$\sigma$ - - standard deviation.

Then the formula of $k$ fold convolution function of $F(t)$ in $T_{X}$ is

$$
\begin{array}{r}
F^{(k)}\left(T_{X}\right)=\Phi\left(\frac{T_{X}-A_{k}}{B_{k}}\right) \\
F^{(k+1)}\left(T_{X}\right)=\Phi\left(\frac{T_{X}-A_{k+1}}{B_{k+1}}\right)
\end{array}
$$

Where $A_{k}=\sum_{i=0}^{k} A_{i}=k \mu, B_{k}=\sigma \sqrt{k}$.

By formula (3), $P(k)$ can be calculated as follows.

$$
P(k)=\Phi\left(\frac{T_{X}-A_{k}}{B_{k}}\right)-\Phi\left(\frac{T_{X}-A_{k+1}}{B_{k+1}}\right)
$$

By formula (4), $P(k \leq N)$ can be calculated as follows.

$$
P(k \leq N)=\sum_{k=0}^{N}\left[\Phi\left(\frac{T_{X}-A_{k}}{B_{k}}\right)-\Phi\left(\frac{T_{X}-A_{k+1}}{B_{k+1}}\right)\right]
$$

(3) The life of unit obeys Waybill distribution

When the life of unit obeys Waybill distribution, the fault density function and the cumulative failure distribution function of the unit can be calculated as follows.

$$
\begin{gathered}
f(t)=\lambda \beta t^{\beta-1} \exp \left(-\lambda t^{\beta}\right) \\
F(t)=1-\exp \left(-\lambda t^{\beta}\right)
\end{gathered}
$$

Where,

$\beta$ - Shape parameter;

$\lambda$ - Scale parameter.

Then the formula of $k$ fold convolution function of $F(t)$ in $T_{X}$ is

$$
F^{(k)}\left(T_{X}\right)=\sum_{n=1}^{\infty} \frac{(-1)^{n-1} A_{k, n} T_{X}^{n \beta}}{\Gamma(n \beta+1)}
$$




$$
F^{(k+1)}\left(T_{X}\right)=\sum_{n=1}^{\infty} \frac{(-1)^{n-1} A_{k+1, n} T_{X}^{n \beta}}{\Gamma(n \beta+1)}
$$

Where, $A_{k, n}$ can be calculated by the following method.

For all $n, A_{1, n}=a_{n}$, when $n<k, A_{k, n}=0$.

For all $k, A_{k, 1}=(-1)^{k-1} a_{k}$, when $n>k, A_{k, n}=-\sum_{j=k-1}^{n-1} A_{k-1, j} a_{n-j}, a_{k}=\Gamma[k \beta+(1 / k !)]$.

By formula (3), $P(k)$ can be calculated as follows.

$$
P(k)=\sum_{n=1}^{\infty} \frac{(-1)^{n-1} A_{k, n} T_{X}^{n \beta}}{\Gamma(n \beta+1)}-\sum_{n=1}^{\infty} \frac{(-1)^{n-1} A_{k+1, n} T_{X}^{n \beta}}{\Gamma(n \beta+1)}
$$

By formula (4), $P(k \leq N)$ can be calculated as follows.

$$
P(k \leq N)=\sum_{k=0}^{N}\left[\sum_{n=1}^{\infty} \frac{(-1)^{n-1} A_{k, n} T_{X}^{n \beta}}{\Gamma(n \beta+1)}-\sum_{n=1}^{\infty} \frac{(-1)^{n-1} A_{k+1, n} T_{X}^{n \beta}}{\Gamma(n \beta+1)}\right]
$$

Formula (22) is obviously an infinite series. It is very difficult to determine the demand for maintenance equipment directly from the formula. So the solution to the problem is using genetic algorithm or simulation method generally.

\subsection{The forecasting model of demand under conditions of multiple unit}

The problem that calculating support probability of the maintenance equipment in multiple units can be solved based on the forecasting model of demand under conditions of single unit by considering the system contains $m$ units in the same type which connected in series.

According to the characteristics of series system, $P(k \leq N)$ can be calculated as follows.

$$
P(k)=\sum_{k=k_{1}+k_{2}+\cdots+k_{m}}\left[P\left(k_{1}\right)+P\left(k_{2}\right)+\cdots+P\left(k_{m}\right)\right]
$$

By formula (22), $P(k \leq N)$ can be calculated as follows.

$$
P(k \leq N)=\sum_{k=0}^{N} P(k)=\sum_{k=0}^{N} \sum_{k=k_{1}+k_{2}+\cdots+k_{m}}\left[P\left(k_{1}\right)+P\left(k_{2}\right)+\cdots+P\left(k_{m}\right)\right]
$$

(1) The life of unit obeys the exponential distribution

According to the characteristic of exponential distribution, the formula for calculating the support probability of the maintenance equipment is

$$
P(k \leq N)=\sum_{k=0}^{N} \frac{\left(m \lambda T_{X}\right)^{k}}{k !} \exp \left(-m \lambda T_{X}\right)
$$

But for the normal distribution and Waybill distribution, when $m$ and $N$ are large, it is hard to solve the problem with formula (24), so it is necessary to simplify.

(2) The life of unit is in normal distribution

The formula for calculating the support probability of the maintenance equipment under the assumption condition is

$$
P(k \leq N)=\sum_{k=0}^{N} C_{m}^{k}\left\{\int_{0}^{T_{X}} \frac{1}{\sigma \sqrt{2 \pi}} \exp \left[-\frac{(x-\mu)^{2}}{2 \sigma^{2}}\right] \mathrm{d} x\right\}^{k} \times\left\{\int_{T_{X}}^{\infty} \frac{1}{\sigma \sqrt{2 \pi}} \exp \left[-\frac{(x-\mu)^{2}}{2 \sigma^{2}}\right] \mathrm{d} x\right\}^{m-k}
$$

(3) The life of unit obeys Waybill distribution

The formula for calculating the support probability of the maintenance equipment under the assumption condition is 


$$
\begin{aligned}
P(k \leq N) & =\sum_{k=0}^{N} C_{m}^{k}\left[1-\exp \left(-\lambda T_{X}^{\beta}\right)\right]^{k}\left[\exp \left(-\lambda T_{X}^{\beta}\right)\right]^{m-k} \\
& =\sum_{k=0}^{N} \sum_{i=0}^{k} C_{m}^{k} C_{k}^{i}(-1)^{i} \exp \left(-i \lambda T_{X}^{\beta}\right)\left[\exp \left(-\lambda T_{X}^{\beta}\right)\right]^{m-k} \\
& =\sum_{k=0}^{N} \sum_{i=0}^{k} C_{m}^{k} C_{k}^{i}(-1)^{i} \exp \left[-(m-n+i)\left(\lambda T_{X}^{\beta}\right)\right]
\end{aligned}
$$

\section{Conclusions}

Prediction of demand for maintenance equipment is one of the basic work of development of the supply standard. On the basis of analyzing the influencing factors of the demand for initial maintenance equipment, The model of forecasting demand for maintenance equipment were established based on the support probability of the maintenance equipment under the condition of different life distribution of units. And the feasibility and validity of the model were verified. This article can provide the theoretical basis for the development of the initial maintenance equipment supply standard. But due to the limited of simulation method, how to improve the accuracy of the model will be the focus of the next step.

\section{References}

[1] NIE Tao. SHENG Wen. Research on two-echelon supply support optimizing for repairable spare parts of K:N system[J]. Systems Engineering and Electronics, 2010,32(7):1452-1455.

[2] HUANG Zhaoxie. Research on Construction Methods for Ordnance Materials Catalog and Standard System[D]. Shijiazhuang:Ordnance Engineering College,2013.

[3] Kenndey W. J., Patterson J. W., Fredendall L. D.. An Overview of Recent Literature on Spare Parts Inv- entories[J]. Int. J. Production Economics, 2002,7:201-215.

[4] Marseguerra M, Zio E, Prodofillini L. Multi-objective Spare Part Allocation by Means of Genetic Algorithms and Monte Carlo Simulation[J]. Reliability Engineering and System Safety,2005, 8:325-335.

[5] Army Needs to Evaluate Impact of Recent Actions to Improve Demand Forecasts for Spare parts[R]. U.S. General Accounting Office-01-772,2009:9-20.

[6] Army Materiel Maintenance Policy[R]. AR750-1.2005,7:23-25.

[7] Bachman T.C. Forecasting Demand for Weapon System Items[R]. ADAD285408,1994:13-15.

[8] LONG Jun, KANG Rui, KANG Xiaoming. Optimization of spares supply based on integrated supply performance measure[J]. Systems Engineering and Electronics,2007,29:2085-2087.

[9] Using Unit Supply System[S]. PAM701-2-1,1997,12:65-67. 